\title{
REVUE GÉNÉRALE
}

\section{La kinésithérapie respiratoire dans la bronchiolite virale aiguë du nourrisson. Arguments pour/contre}

\section{Respiratory physiotherapy in acute viral bronchiolitis in the newborn. Pro/con arguments}

\author{
G. Postiaux ${ }^{a}, *$, P. Maffei ${ }^{b}$, J.-C. Villiot-Danger ${ }^{c}$, \\ J.-C. Dubus ${ }^{\mathrm{d}}$
}

a Groupe d'étude pluridisciplinaire stéthacoustique, services des soins intensifs, de médecine interne et de pédiatrie, grand hôpital de Charleroi (GHDC), 6000 Charleroi, Belgique b Pôle de médecine physique et de réadaptation, hôpital de la Conception, Assistance publique des Hôpitaux de Marseille, 147, boulevard Baille, 13385 Marseille cedex 5, France

c 10, avenue du Général-Barbot, 05100 Briançon, France

d Médecine infantile, pneumo-allergologie et CRCM \& CNRS, URMITE 6236, Assistance publique-Hôpitaux de Marseille, 13385 Marseille cedex 5, France

Reçu le 8 mars 2017 ; accepté le 30 septembre 2017

Disponible sur Internet le 10 mai 2018

\section{MOTS CLÉS}

Bronchiolite ;

Nourrisson ;

Kinésithérapie

respiratoire
Résumé Cet article rapporte un échange d'arguments objectifs entre Mr Guy Postiaux plaidant pour la kinésithérapie respiratoire dans la bronchiolite vitale aiguë du nourrisson et le $\operatorname{Pr}$ Jean-Christophe Dubus plaidant contre. Il ressort de la revue de la littérature que les méthodes usuelles de kinésithérapie doivent être abandonnées parce qu'elles ne sont pas validées et peuvent présenter des effets secondaires délétères. La plus récente révision Cochrane (2016) suggère l'utilisation des techniques expiratoires lentes qui possèdent des éléments de validation et qui ne présentent aucun effet secondaire délétère. De larges études multicentriques doivent

\footnotetext{
* Auteur correspondant. Boulevard Joseph-II n 4 B 71, B, 6000 Charleroi, Belgique. Adresse e-mail : guy.postiaux@gmail.com (G. Postiaux).
} 


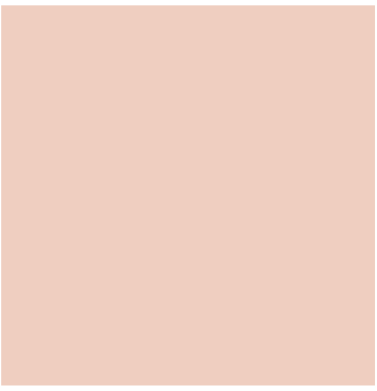

être réalisées pour confirmer ou infirmer les résultats des cinq études retenues par la Cochrane. Leur analyse permet d'extraire des éléments objectifs attestant de l'efficacité des techniques expiratoires lentes sur la désobstruction bronchopulmonaire et sur la réduction de son degré de sévérité à court et moyen termes. Les études concernant la kinésithérapie respiratoire en ambulatoire pour les bronchiolites de gravité modérée ne nécessitant pas l'hospitalisation manquent. Une évaluation fondée sur la physiopathologie d'une obstruction bronchique multifactorielle et sur la sémiologie dont l'auscultation représente la pierre angulaire est nécessaire. (c) 2018 SPLF. Publié par Elsevier Masson SAS. Tous droits réservés.

\section{KEYWORDS}

Bronchiolitis; Newborn; Respiratory physiotherapy

\section{Abréviations}

AFE accélération/augmentation du flux expiratoire ANP aspiration nasopharyngée au moyen d'une sonde BVA bronchiolite virale aiguë cCPT conventional chest physical therapy (FET + clapping + drainage postural)

DAA drainage autogène assisté

DDB dilatation des bronches

DRR désobstruction rhinopharyngée rétrograde ou DRP désobstruction rhinopharyngée

ELPr expiration lente prolongée

FET forced expiration technique-technique de l'expiration forcée

$\mathrm{FIO}_{2}$ fraction d'oxygène dans l'air inspiré

$\mathrm{FR}$ fréquence respiratoire

KR kinésithérapie respiratoire

NEB nébulisation

NICE National Institute for Health and Care Excellence

OMA otite moyenne aiguë

RGO reflux gastro-œsophagien

SW score de Wang

TP toux provoquée

VAD voies aériennes distales

VEMS volume expiré maximum en une seconde

VPI ventilation à percussion intrapulmonaire

Wh \% taux de sibilances (Wh \% : wheezing rate)

\section{Introduction}

Lors du congrès organisé à Marseille le 26 novembre 2016 à l'initiative de la SKR-Société de kinésithérapie de réanimation, le Pr Jean-Christophe Dubus (JCD) et Mr Guy Postiaux (GP) ont présenté l'argument invalidant ou validant la kinésithérapie respiratoire $(K R)$ dans la bronchiolite virale aiguë du nourrisson (BVA). Ce débat est récurrent mais de récentes publications autorisent une « remise à plat » de cette question alors que de nombreuses conférences de consensus étrangères ne recommandent plus son utilisation dans cette indication. L'échange des arguments se base sur des éléments objectifs tirés de publications référencées qui font état de trois méthodes de KR retenues par la plus récente révision Cochrane (2016) : la conventional Chest Physical Therapy (cCPT), l'accélération du flux expiratoire (AFE) et l'expiration lente prolongée (ELPr) [1].

\section{Arguments contre : Pr Jean-Christophe Dubus}

\section{Les recommandations des sociétés savantes}

Les recommandations françaises de l'ANAES datant de plus de 15 ans accordaient valeur à la KR dans la BVA. Il s'agissait d'un avis d'expert de grade C [2]. Les recommandations plus récentes sont différentes. Ainsi, la KR n'est pas 
recommandée aux États-Unis (recommandation de grade $\mathrm{B}$, force modérée) [3]. Il en est de même en Italie [4]. En Grande Bretagne, la KR n'est recommandée qu'en présence de comorbidités telles qu'une trachéomalacie ou une amyotrophie spinale.

Les publications successives de la revue Cochrane des années 2005, 2007 et 2012 ainsi que la dernière mise à jour publiée en février 2016 concluent au fait qu' « Aucune des techniques analysées de KR n'a montré de réduction de la sévérité de la BVA. Pour ces raisons, ces techniques ne doivent pas être utilisées en pratique courante chez les patients hospitalisés ».

Si les techniques de désobstruction bronchique sont efficaces dans la mucoviscidose et les dilatations des bronches (DDB), force est d'admettre que ces principes ont été extrapolés aux patients atteints de BVA et de pneumonie dans un but de désobstruction et de lutte contre les complications (atélectasies, surinfection, hypoxémie...). Cependant, dans la BVA, les études cliniques sont rares. La confusion entre différentes techniques de KR utilisées (percussions/vibrations, augmentation/accélération du flux expiratoire (seule ou avec toux provoquée) et l'ELPr rendent l'interprétation des études difficile.

\section{Diagnostic et physiopathologie de la BVA}

La BVA se définit soit comme un premier épisode respiratoire à prédominance expiratoire, qui concerne un enfant de moins de 1 an dans les pays anglo-saxons, soit comme 1 ou 2 épisodes de ce type chez un enfant de moins de 2 ans en France. On en dénombre 500000 cas par hiver en France. L'agent pathogène le plus fréquent est le virus respiratoire syncytial. L'obstruction est bronchiobronchiolaire. Il s'agit d'une obstruction mécanique liée à des débris cellulaires (nécrose de l'épithélium par le virus), une hypersécrétion, un œdème péribronchiolaire. La part de bronchoconstriction est modérée, voire nulle. Cette obstruction peut s'accompagner d'hypoxie, d'emphysème, d'atélectasies.

\section{Traitement de la BVA}

Le traitement est avant tout symptomatique. Il consiste en lavages de nez, hydratation/alimentation, administration d'oxygène si nécessaire, précautions environnementales. La KR est proposée de façon variable selon les pays [5,6] : prescrite dans environ $60 \%$ des cas en France, alors qu'elle n'est que de 13 à $4 \%$ au Canada et aux États-Unis. Elle n'est pas non plus réalisée dans cette indication en Suisse. Pourtant, les indices de morbi-mortalité sont superposables entre ces différents pays (Tableau 1). Une séance de KR débute par les soins de la sphère ORL, mais quels sont les niveaux de preuve d'efficacité de celle-ci ? Les recommandations sont de grade $A$ : le lavage de nez réduit la production de cytokines (parasinus) ainsi que la durée de la maladie. Mais les modalités en sont mal précisées. La question de la réalisation de l'acte par un personnel de santé est cependant posée en raison de la mauvaise connaissance parentale du lavage de nez avec seulement $21 \%$ d'entre eux sachant le réaliser [7]. Faut-il pour autant que ce soin soit réalisé par un kinésithérapeute ? Cela pose question en raison du possible coût induit. En ce qui concerne la KR elle-même, les études disponibles utilisent des critères différents et ne sont pas exemptes de biais (Tableau 2). Une étude (parue en juin 2016 [8]) non encore reprise dans la revue Cochrane (parue en février 2016) sur un échantillon de 93 enfants (âge $<2$ ans) atteints de bronchiolite compare trois techniques : le drainage autogène assisté (DAA), la ventilation à percussion intrapulmonaire (VPI) et le «bouncing » (vaet-vient sur ballon). Les résultats montrent des délais de guérison significativement différents au profit du DAA vs la VPI (3,6 $\pm 1,4$ jours vs 4,5 $\pm 1,9$ jours $)$ ainsi qu'une amélioration du score de Wang (SW) pour ces deux techniques [9]. Il n'est pas mentionné si un lavage de nez préalable est réalisé, l'étude n'évalue pas les durées d'hospitalisation ni ne fait état des problèmes de coût et de désinfection de l'appareillage de percussions intrapulmonaires.

\section{Situations particulières}

Au total, les indications de la KR sont modestes au regard de la littérature, mais peut-être pourrait-elle se justifier dans des formes particulières de BVA avec pneumonie ou requérant une admission en unité de soins intensifs pédiatriques (USIP) ?

\section{En présence d'un foyer radiologique (pneumonie)}

Il n'y a aucune étude s'intéressant spécifiquement à l'intérêt de la KR dans les bronchiolites avec foyer radiologique. Les seules données disponibles sont celles de la KR dans le cadre des pneumonies communautaires de l'enfant. L'étude de Paludo n'observe qu'une réduction de la durée de la toux, sans amélioration clinique, mais une amélioration des signes stéthacoustiques [10]. L'étude de Lukrafka et al. n'observe aucune amélioration du score clinique ni de la durée d'hospitalisation [11]. Une revue Cochrane de 2013 colligeant trois études randomisées et contrôlées incluant un total de 255 patients conclut à l'inefficacité de la $K R$ en raison de la rareté des études et du manque de données [12]. Elle note cependant un effet positif sur la fréquence respiratoire et la saturation dans deux études sur trois.

\section{En réanimation ou USIP}

L'étude de Barnard-Narbonne portant sur 20 nourrissons ventilés pour bronchiolite rapporte que l'expiration lente prolongée améliore les paramètres de la ventilation et la $\mathrm{FlO}_{2}$. Cette étude présente cependant des biais : la faiblesse de l'échantillon, l'absence de suivi et d'évaluation des durées d'hospitalisation et de la ventilation [13]. Une autre étude rétrospective menée dans le Nord-Est des ÉtatsUnis et portant sur 323 enfants avec bronchiolite à VRS note la variation des prises en charge, le taux d'intubation variant d'un facteur 3,5, la KR et le salbutamol étant les deux traitements les plus prescrits [14]. L'auteur observe une relation directe entre une durée de séjour allongée et certains traitements, notamment la ventilation, les corticoïdes inhalés, la VIP et la KR. Il n'a pas fait mention de gravité ou de complications. Il n'y a en tout cas pas de réduction de la durée d'hospitalisation des enfants sous nébulisation ou KR. 
Tableau 1 Données d'hospitalisation et mortalité liées à la bronchiolite du nourrisson (d'après [5]).

\begin{tabular}{lllll}
\hline & Âge des enfants & Taux d'hospitalisation & Durée de séjour (jours) & Mortalité \\
\hline France $(2009)$ & Moins de 1 an & $35,8 / 1000$ & 4,3 & $2,6 / 100000$ \\
Angleterre $(2002,2003)$ & Moins de 2 ans & $30,8 / 1000$ & 2,7 & $2,4 / 100000$ \\
États-Unis (2007, 2009) & Moins de 1 an & $23,5 / 1000$ & 2,4 & $2,4 / 100000$ \\
\hline
\end{tabular}

Tableau 2 Études publiées sur la kinésithérapie respiratoire dans la bronchiolite aiguë du nourrisson (tiré de [5]).

\begin{tabular}{|c|c|c|c|c|c|c|c|}
\hline Étude & Technique & $\begin{array}{l}\text { Critère de } \\
\text { jugement }\end{array}$ & $\begin{array}{l}\text { Nombre } \\
\text { d'enfants }\end{array}$ & $\begin{array}{l}\text { Lieu de } \\
\text { l'étude }\end{array}$ & Résultats & Type d'études & Commentaires \\
\hline Webb [9] & PV & $\begin{array}{l}\text { Durée } \\
\text { d'évolution }\end{array}$ & 90 & $\begin{array}{l}\text { Hôpital, } \\
\text { Angleterre }\end{array}$ & $\begin{array}{l}\text { Pas de } \\
\text { différence }\end{array}$ & Prospective & $\begin{array}{l}\text { Technique } \\
\text { inappropriée }\end{array}$ \\
\hline Ralston [19] & PV & $\begin{array}{l}\text { Utilisation de } \\
\text { la KR }\end{array}$ & 11568 & $\begin{array}{l}\text { Hôpitaux, } \\
\text { États-Unis }\end{array}$ & $\begin{array}{l}\text { Diminution de } \\
14 \text { à } 4 \%\end{array}$ & Prospective & Observationnelle \\
\hline Gajdos [20] & $\begin{array}{l}\mathrm{AF}+\text { toux } \\
\text { provoquée }\end{array}$ & $\begin{array}{l}\text { Délai de } \\
\text { guérison }\end{array}$ & 496 & $\begin{array}{l}\text { Hôpitaux, } \\
\text { France }\end{array}$ & $\begin{array}{l}\text { Évolution idem } \\
\text { Confort } \\
\text { ressenti } \\
\text { Idem }\end{array}$ & Prospective & $\begin{array}{l}\text { Atélectasie } \\
10 \%\end{array}$ \\
\hline Rochat [22] & $\begin{array}{l}\text { ELP } \\
A F\end{array}$ & $\begin{array}{l}\text { Stabilité } \\
\text { clinique }\end{array}$ & 99 & $\begin{array}{l}\text { Hôpital, } \\
\text { Genève }\end{array}$ & $\begin{array}{l}\text { Pas de } \\
\text { différence }\end{array}$ & Monocentrique & $\begin{array}{l}\text { Pas de réelle } \\
\text { durée } \\
\text { d'hospitalisation }\end{array}$ \\
\hline Sanchez [23] & $\begin{array}{l}\text { ELP AF + toux } \\
\text { provoquée }\end{array}$ & $\begin{array}{l}\text { Durée } \\
\text { d'hospitalisation } \\
\text { et d'utilisation } \\
\text { de l'oxygène }\end{array}$ & 236 & $\begin{array}{l}\text { Hôpital, } \\
\text { Espagne }\end{array}$ & $\begin{array}{l}\text { Pas de } \\
\text { différence }\end{array}$ & Double insu & $\begin{array}{l}\text { Biais: } \\
\text { utilisation de } \\
\text { broncho- } \\
\text { dilatateurs, } \\
\text { Corticoïdes... }\end{array}$ \\
\hline Pupin [26] & $\begin{array}{l}\text { ELP } \\
\text { PV placebo }\end{array}$ & $\begin{array}{l}\mathrm{SpO}_{2}, \\
\text { fréquence } \\
\text { respiratoire et } \\
\text { cardiaque }\end{array}$ & 81 & $\begin{array}{l}\text { Hôpital, } \\
\text { Brésil }\end{array}$ & $\begin{array}{l}\text { Pas de } \\
\text { différence }\end{array}$ & Monocentrique & $\begin{array}{l}\text { Critères } \\
\text { cliniques } \\
\text { Petite cohorte }\end{array}$ \\
\hline Cornes [27] & $\begin{array}{l}\text { ELP + déglutition } \\
\text { Score de Wang } \\
\text { rétrograde PV } \\
\text { DRP(AMP) }\end{array}$ & Score de Wang & 30 & $\begin{array}{l}\text { Hôpital, } \\
\text { Brésil }\end{array}$ & $\begin{array}{l}\text { Amélioration à } \\
\text { j3 dans le } \\
\text { groupe ELP }\end{array}$ & Monocentrique & $\begin{array}{l}\text { Effectifs } \\
\text { réduits } \\
\text { Score clinique }\end{array}$ \\
\hline Postiaux [30] & SSH SSH+ELP & $\begin{array}{l}\text { Score de Wang } \\
\mathrm{SpO}_{2}\end{array}$ & 20 & $\begin{array}{l}\text { Hôpital, } \\
\text { Belgique }\end{array}$ & $\begin{array}{l}\text { Diminution du } \\
\text { score de Wang }\end{array}$ & Monocentrique & $\begin{array}{l}\text { Effectifs } \\
\text { réduits } \\
\text { Pas de durée } \\
\text { d'évolution }\end{array}$ \\
\hline
\end{tabular}

\section{Et en ville?}

Une étude réalisée dans l'Essonne en 2006 portant sur 407 nourrissons traités par AFE et toux provoquée (TP) ne note pas de différence après traitement sur la $F R$, avec $2 / 3$ des patients gardant une gêne respiratoire et un score clinique obtenu d'autant moins bon que l'enfant est jeune [15].

\section{KR et effets latéraux}

Dans l'étude multicentrique réalisée en France et portant sur 496 enfants hospitalisés (étude BRONKINOU) de sérieux effets secondaires délétères sont constatés dans le groupe AFE + aspiration nasale (AN) : bradycardie avec désaturation, bradycardie sans désaturation, vomissements durant la procédure (Fig. 1), déstabilisation respiratoire et hypotonie [16] (Tableau 3). D'autres effets secondaires ont été rapportés comme des fractures de côte, à différencier d'une maltraitance, par Chanelière et al. [17], Chalumeau et al. [18], et Gorincour et al. [19] (Fig. 2) ainsi que des épisodes de reflux gastro-œsophagien (RGO) [20]. Même l'aspiration nasopharyngée (ANP) est battue en brèche, une étude brésilienne montrant une tolérance de l'ANP bien moins bonne que la DRR, [21] chez 100 enfants (âge $<12$ mois) avec bronchiolite (Tableau 4). Ainsi, depuis 


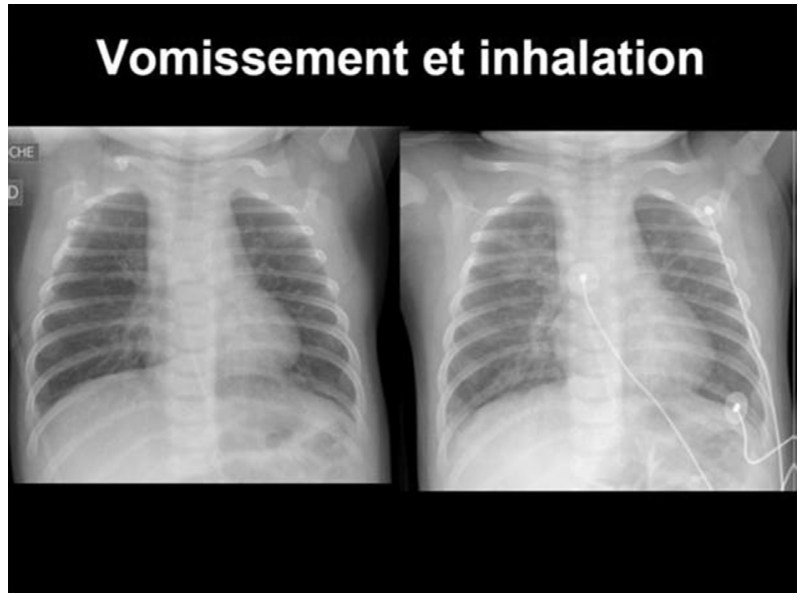

Figure 1. Inhalation de matériau alimentaire visible au niveau de la bronche lobaire inférieure droite.
2015, les recommandations du National Institute for Health and Care Excellence (NICE) sont les suivantes : " l'ANP n'est à considérer qu'en présence de détresse respiratoire ou de difficultés alimentaires en rapport avec les sécrétions des voies aériennes supérieures ou en présence d'apnée ».

\section{Conclusion du premier exposé «contre»}

Les effets de la KR dans la BVA sont peu probants. On manque d'études, par exemple sur les enfants avec toux inefficace ou atélectasie, et les effets collatéraux de la KR restent possibles.

- Arguments contre : globalement, les effets de la KR dans la BVA sont peu probants et, par exemple, on manque d'études sur les enfants avec toux inefficace ou atélectasie ; de plus, on ne peut pas exclure des effets secondaires de la KR.

Tableau 3 Effets secondaires délétères de la kinésithérapie respiratoire par accélération du flux expiratoire (d'après [16] avec permission).

\begin{tabular}{lllll}
\hline & Non traités $n=250$ & Traités $(n=246)$ & Risque relatif $($ IC $95 \%)$ & Test de Fischer $(p)$ \\
\hline Bradycardie sans désaturation, $n(\%)$ & $3(1,2 \%)$ & $3(1,2 \%)$ & $1,0(0,2-5,0)$ & 1,00 \\
Bradycardie avec désaturation, $n(\%)$ & $2(0,8 \%)$ & $7(2,8 \%)$ & $3,6(0,7-16,9)$ & 0,10 \\
Vomissements pendant la séance, $n(\%)$ & $1(0,4 \%)$ & $10(4,1 \%)$ & $10,2(1,3-78,8)$ & 0,005 \\
Dégradation respiratoire, $n(\%)$ & $3(1,2 \%)$ & $16(6,5 \%)$ & $5,4(1,6-8,4)$ & 0,002 \\
Hyopotonie, $n(\%)$ & 0 & $2(0,8 \%)$ & Non calculable & 0,24
\end{tabular}

a.

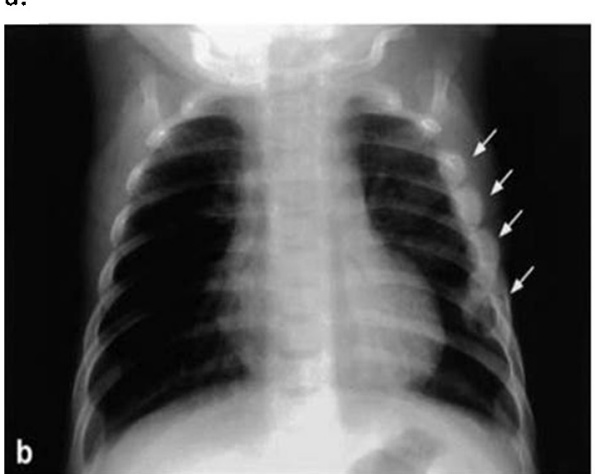

b.

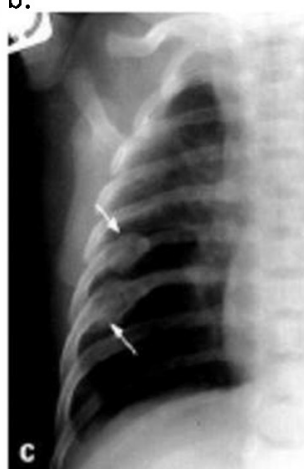

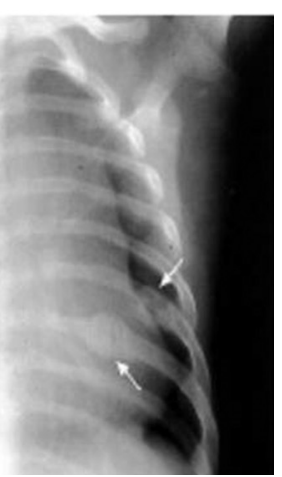

Figure 2. Fractures de côtes après kinésithérapie au moyen de l'AFE. Le cliché révèle 12 fractures/14 à la partie latérale du thorax de la $4^{\mathrm{e}}$ à la $7^{\mathrm{e}}$ côte.

Chalumeau, Pediatr Radiol 2002;32;644-7. (Avec la permission de Springer Verlag).

Tableau 4 Comparaison entre aspiration (ASP) et reniflement (REN) selon le degré de sévérité légère, modérée ou sévère dans la bronchiolite aiguë (d'après [21]).

\begin{tabular}{|c|c|c|c|c|c|c|}
\hline & \multicolumn{2}{|l|}{ Lègère } & \multicolumn{2}{|c|}{ Modérée } & \multicolumn{2}{|l|}{ Grave } \\
\hline & $\begin{array}{l}\text { ASP } \\
(n=2)\end{array}$ & $\begin{array}{l}\text { REN } \\
(n=2)\end{array}$ & $\begin{array}{l}\text { ASP } \\
(n=29)\end{array}$ & $\begin{array}{l}\text { REN } \\
(n=26)\end{array}$ & $\begin{array}{l}\text { ASP } \\
(n=11)\end{array}$ & $\begin{array}{l}\text { REN } \\
(n=12)\end{array}$ \\
\hline Tirage thoracique & 50,0 & 50,0 & 100,0 & $84,6 *$ & 100,0 & 100,0 \\
\hline Saignement nasal & 50,0 & 0 & 44,8 & $0 *$ & 63,6 & $8,3^{*}$ \\
\hline Wheezing & 0 & 50,0 & 62,1 & 46,2 & 36,4 & 75,0 \\
\hline
\end{tabular}


- Il y a plus de 15 ans que l'ANAES recommandait la KR dans la BVA, mais de nos jours la KR n'est plus recommandée aux États-Unis, en Italie et en Grande Bretagne, si ce n'est en présence de comorbidités telles qu'une trachéomalacie ou une amyotrophie spinale.

- Selon la revue Cochrane, « ces techniques ne doivent pas être utilisées en pratique courante chez les patients hospitalisés ». La KR est efficace pour la désobstruction bronchique dans la mucoviscidose et la dilatation des bronches, mais cela ne peut pas être extrapolé à la BVA, dans laquelle les études cliniques sont rares.

- Le traitement de la BVA est avant tout symptomatique (lavages de nez, hydratation/alimentation, éventuellement oxygénothérapie) ; la KR est souvent proposée en France mais rarement au Canada, aux États-Unis et en Suisse, avec une morbi-mortalité superposable entre ces différents pays.

- La KR pourrait peut-être être justifiée dans des formes particulières de BVA avec pneumonie ou requérant une admission en unité de soins intensifs pédiatriques (USIP).

- En présence d'un foyer radiologique (pneumonie), une étude a montré que la KR diminuait la durée de la toux et améliorait les signes stéthacoustiques, sans amélioration clinique et d'autres études ont conclu à l'inefficacité de la KR.

- En réanimation ou USIP, une étude a rapporté que l'expiration lente prolongée améliorait les paramètres de la ventilation et la $\mathrm{FIO}_{2}$, mais elle était entachée de biais et une autre étude prospective n'a pas rapporté de réduction de la durée d'hospitalisation des enfants sous nébulisation ou KR.

- En ville, une étude portant sur 407 nourrissons traités par AFE et toux provoquée (TP) n'a pas rapporté de différence après traitement sur la $F R$, avec $2 / 3$ des patients gardant une gêne respiratoire ; le score clinique obtenu était d'autant moins bon que l'enfant était jeune.

- Plusieurs études ont rapporté des effets secondaires délétères de la KR : bradycardie avec ou sans désaturation, vomissements durant la procédure, déstabilisation respiratoire, hypotonie, fractures de côte, reflux gastro-œsophagien.

- Même, l'intérêt de l'aspiration nasopharyngée (ANP) a été remis en cause et, depuis 2015, le National Institute for Health and Care Excellence signale que « l'ANP n'est à considérer qu'en présence de détresse respiratoire ou de difficultés alimentaires en rapport avec les sécrétions des voies aériennes supérieures ou en présence d'apnée ».

\section{Arguments pour : Mr Guy Postiaux}

À l'entame de l'argumentaire pour, il convient de rappeler que la KR est symptomatique et non pas nosologique, ce qui signifie que, quelle qu'en soit la cause, c'est le caractère sémiologique de l'obstruction bronchopulmonaire qui doit être pris en compte. Quelques notions de physiopathologie viennent également à l'appui de l'analyse.

\section{Voies aériennes intrathoraciques}

La dernière révision Cochrane [1] qui fait autorité a tenu compte des critiques adressées aux versions précédentes portant sur l'amalgame et la confusion entre les méthodes de KR et sur l'absence de prise en compte du degré de gravité de la BVA $[22,23]$. Elle distingue pour la première fois cCPT, AFE et ELPr.

\section{La conclusion comporte trois sections}

A. La première section établit « qu'aucun des trois modes d'intervention n'a montré d'amélioration significative chez les patients hospitalisés atteints de bronchiolite sévère ».

Commentaire : on ne peut qu'adhérer à cette analyse conforme aux recommandations des sociétés savantes qui conviennent de s'abstenir de toute manipulation physique à ce stade de gravité.

B. La seconde section concerne spécifiquement «l'AFE qui entraîne de sérieux effets délétères ».

Commentaires : une étude mécanique publiée en 1992 [24] identifiait le collapsus trachéal généré par cette procédure et montrait le caractère antiphysiologique de l'AFE. Au cours des années qui suivirent d'autres effets secondaires délétères sévères furent mis en avant et dont le premier intervenant dresse la liste (JCD) [15]. Ces effets délétères concernent la forme initiale soudaine et vigoureuse de l'AFE qui pouvait relever d'une forme de brutalité envers les jeunes enfants. En raison d'une littérature critique, cette procédure a par la suite évolué dans ses modalités d'applications d'accélération vers une augmentation, puis vers une augmentation lente et enfin vers la modulation de flux, tendant à rejoindre ainsi un mode expiratoire lent. On ignore quelles réalités fonctionnelles ces termes recouvrent, ce qui rend leur duplication et leur standardisation difficiles.

C. La troisième section concerne l'ELPr qui démontrerait une « amélioration immédiate et transitoire dans la BVA de gravité modérée sans impact sur le décours de la maladie. Des études en ambulatoire sont requises chez les patients infectés par le virus respiratoire syncytial en étudiant l'association de la manœuvre physique à une nébulisation de salbutamol ou de solution saline hypertonique ».

Commentaires : il est fait mention pour la première fois d'une alternative physiothérapique. La Cochrane a colligé quatre publications portant sur un total de 286 cas [25-28]. Deux études sont postérieures à la révision de février 2016. La première réalisée en milieu hospitalier $(n=93)$ porte le nombre total de cas à 379 [7]. La seconde est réalisée en ambulatoire [29], mais elle est affectée d'importants biais méthodologiques qui la rendent difficilement exploitable. Le SW, dont il conviendra d'interpréter la signification, constitue la variable principale des mesures dans les études retenues. 


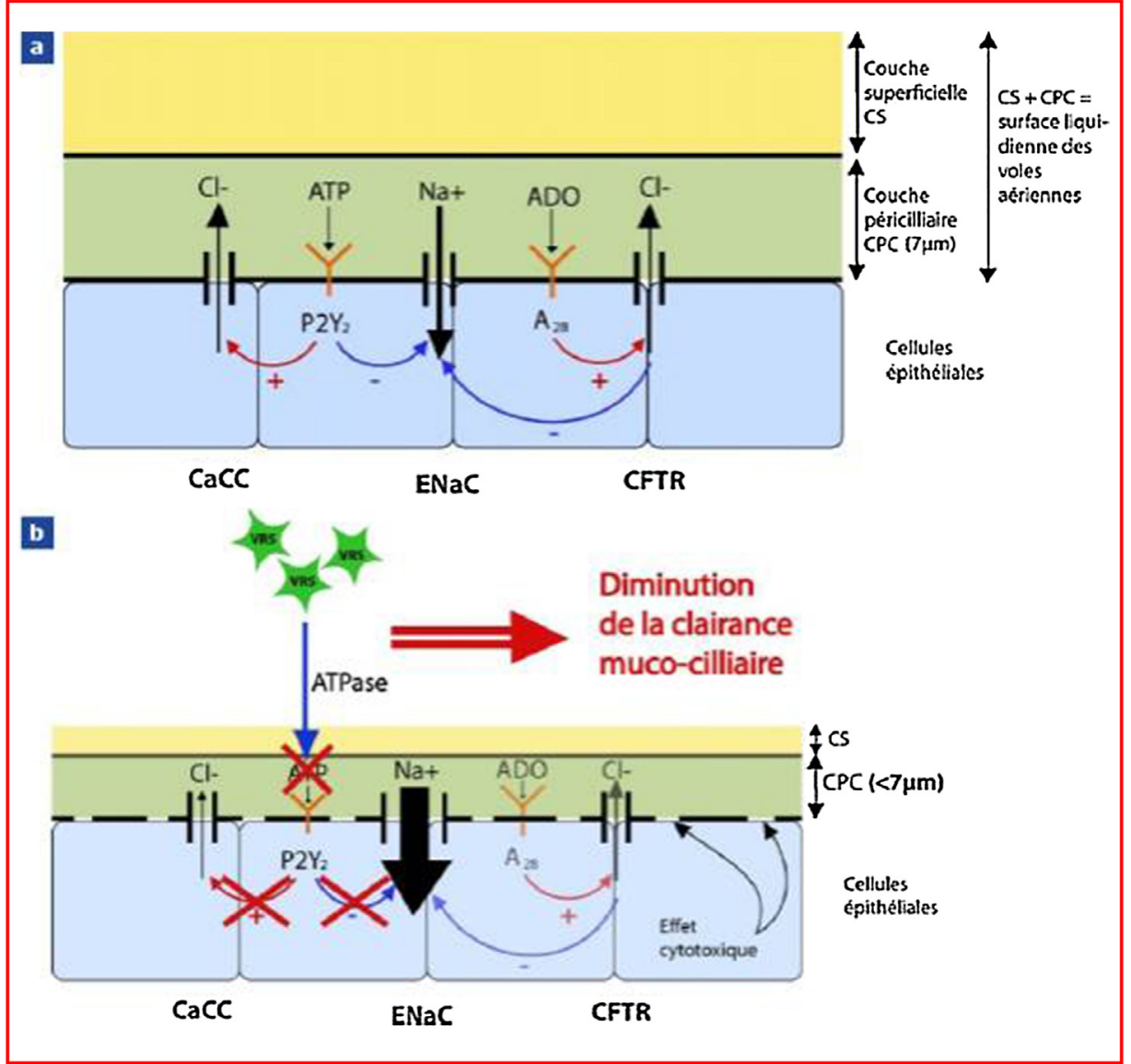

Figure 3. Mécanisme régissant la surface liquidienne des voies aériennes. a : surface liquidienne normale des voies aériennes ; b : mécanismes responsables de la diminution de la clairance mucociliaire lors de la bronchiolite aiguë à virus respiratoire syncytial. (Sauvaget et al. Sérum hypertonique et bronchiolite aiguë du nourrisson. Arch Pediatr 2012;19:635-641). Une nébulisation iso- ou hypertonique est susceptible de réduire l'œdème bronchiolaire et préparer les manœuvres physiques de désobstruction.

Sauvaget E, David M, Bresson V, Retornaz K, Bosdure E, Dubus JC. Sérum salé hypertonique nébulisé et bronchiolite du nourrisson. Arch Pediatr 2012;19:635-41.

\section{La kinésithérapie respiratoire sur une base symptomatique et fonctionnelle}

La KR «dans » la BVA peut être poursuivie à condition de s'appuyer :

- sur la symptomatologie d'une obstruction de type multifactoriel ;

- sur la validité fonctionnelle d'une manœuvre physique adaptée aux particularités mécaniques du système respiratoire du nourrisson ;

- dont les indications ou contre-indications reposent sur la reconnaissance du degré de sévérité clinique de l'obstruction.

Le caractère multifactoriel de l'obstruction bronchique dans la BVA [3] associe œdème, bronchospasme et hypersécrétion affectant les voies aériennes distales-bronchioles (VAD). Cette association définit l'ordre des priorités et justifie, surtout au stade aigu de l'affection, de traiter l'œdème avant de procéder à une manœuvre physique. (Fig. 3) Les études récentes identifient les mécanismes de genèse et les paramètres des bruits adventices, sibilances (résultant de l'œdème et du bronchospasme) et craquements (crépitants) davantage en relation avec la présence de sécrétions dans les VAD. L'auscultation médiate permet ainsi d'apprécier le taux de sibilances (Wh \%) corrélé à l'importance de l'obstruction [30-32]. (Fig. 4) Cependant, chez le petit enfant, une proportion (non définie) de sibilances résulte de la présence d'un encombrement bronchique adhérant susceptible d'être fluidifié par la nébulisation iso- ou hypertonique [33]. La solution saline n'est pas recommandée dans les récentes publications internationales. Elle semble mieux tolérée dans la BVA modérée [34], mais peut présenter de sérieux effets adverses au stade de grande sévérité $[35,36]$. L'intérêt d'une nébulisation réside principalement dans la fluidification et l'hydratation des sécrétions bronchiques hydrophiles qui en facilitent la clairance. La nébulisation devrait précéder les manœuvres physiques.

La validation fonctionnelle des manœuvres expiratoires (Fig. 5) a fait l'objet d'une étude mesurant les composants de l'équation fondamentale du système respiratoire (équation de Rörher-Otis 1919, 1926) [23]. Cette étude établit le caractère physiologique de l'ELPr qui tient compte des caractéristiques mécaniques de l'appareil respiratoire du jeune enfant (trachée compliante, instabilité du thorax, hyperinflation physiologique). L'échec des procédures antérieures de la KR doit en partie être attribué à la 


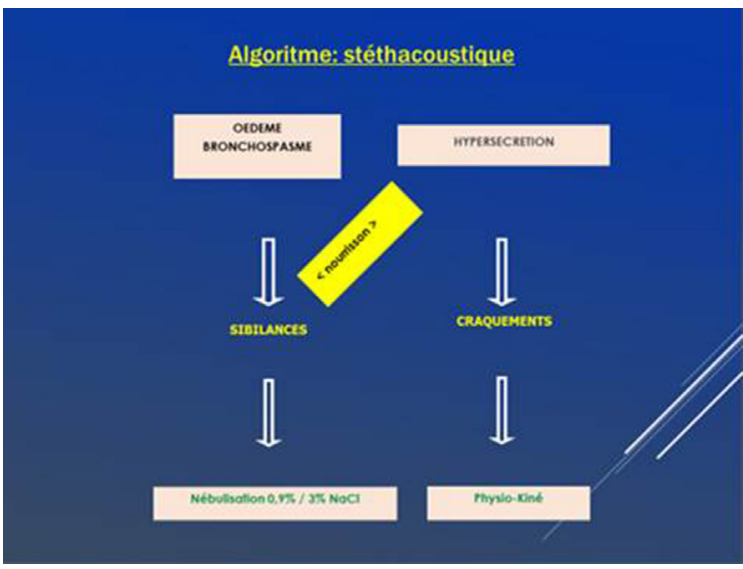

Figure 4. L'obstruction est multifactorielle associant œdème, bronchospasme et hypersécrétion. Les séquences thérapeutiques se fondent sur la présence à l'auscultation des bruits adventices, sibilances et/ou craquements. La kinésithérapie est symptomatique et non pas nosologique.

Kinésithérapie et bruits respiratoires. Nouveau paradigme. Ed. DeboeckSupérieur avril 2016:445.

transposition inappropriée des techniques de l'adulte au jeune enfant. Les manœuvres passives visant à générer des flux s'accompagnent le plus souvent du collapsus de la trachée du nourrisson.

Plusieurs scores permettent d'évaluer le degré de l'obstruction bronchique. Le plus usité est le SW [8] qui est spécifique à la BVA et qui possède une bonne reproductibilité interpersonnelle [37]. Ce score comprend quatre variables gradées de 1 à 3 , soit un résultat total qui va de 0 à 12 ; l'enfant est d'autant plus obstructif que le SW est élevé (Tableau 5). En recoupant les diverses publications ayant eu recours à ce score, il est possible de corréler la valeur du score au degré de gravité. Lorsque le SW est égal ou inférieur à 3, la BVA peut être qualifiée de légère, pour un SW de 4 à 8 de modérée et de sévère au-delà de 8 . La BVA modérée constitue l'indication de la KR [38]. (Fig. 6). La KR est contre-indiquée dans la BVA sévère et inutile dans la BVA légère qui évolue naturellement vers la guérison.

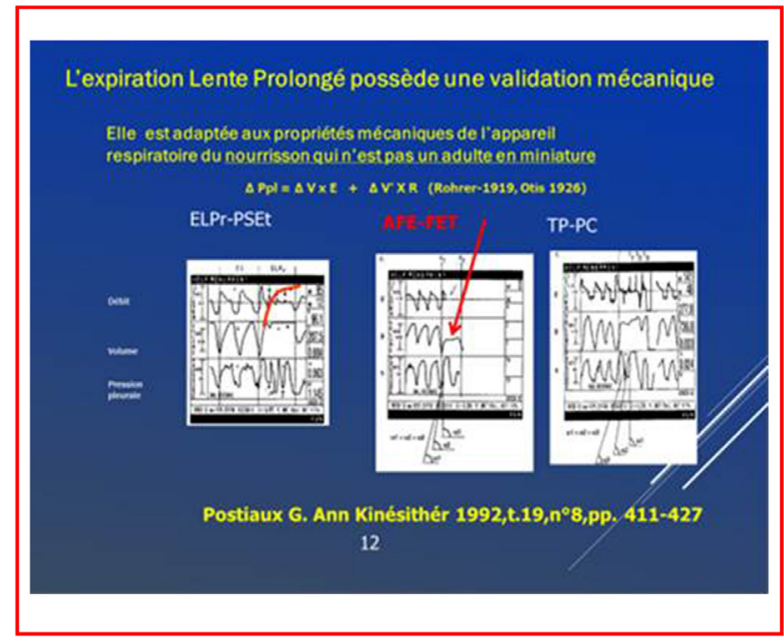

Figure 5. Comparaison entre les comportements mécaniques de l'appareil respiratoire du nourrisson lors de l'ELPr (expiration lente prolongée), l'AFE (accélération du flux expiratoire) et la TP (toux provoquée). Les tracés sont obtenus au moyen de la mesure des éléments constitutifs de l'équation du mouvement du poumon, équation dite de Röhrer-OTIS). La flèche sur la figure indique l'interruption du flux trachéal engendrée par une pression transpulmonaire excessive lors de l'AFE responsable d'un collapsus de la trachée compliante du nourrisson. ELPr : expiration lente prolongée (= PSEt : prolonged slow expiration technique), TP : toux provoquée $(=\mathrm{CP}$ : coughing provoked $)$.

Kinésithérapie et bruits respiratoires. Nouveau paradigme. Ed DeboeckSupérieur 2016:458).

\section{Discussion}

Après 40 années d'errements divers, une fenêtre s'ouvre enfin qui nous apporte quelques éléments de validation des effets à court et moyen termes de la KR « dans » la BVA au moyen d'expirations lentes. La séquence logique d'intervention comprend nébulisation (NEB) + ELPr + désobstruction rhinopharyngée rétrograde (DRR).

Tableau 5 Score de Wang (Wang Clinical Severity Scoring System).

\begin{tabular}{|c|c|c|c|c|}
\hline & \multicolumn{4}{|l|}{ Score } \\
\hline & 0 & 1 & 2 & 3 \\
\hline $\begin{array}{l}\text { Respiratory rate } \\
\text { (breaths/min j) }\end{array}$ & $<30$ & $31-45$ & 4660 & $>60$ \\
\hline Wheezing & None & $\begin{array}{l}\text { Terminal expiratory } \\
\text { or only with } \\
\text { stethoscope }\end{array}$ & $\begin{array}{l}\text { Entire expiration or } \\
\text { audible during } \\
\text { expiration without } \\
\text { stethoscope }\end{array}$ & $\begin{array}{l}\text { Inspiration and expiration } \\
\text { without stethoscope }\end{array}$ \\
\hline $\begin{array}{l}\text { Retractions General } \\
\text { condition }\end{array}$ & None Normal & Intercostal only & Tracheostemal & $\begin{array}{l}\text { Severe with nasal flaring } \\
\text { Irritable, lethargic, poor } \\
\text { Feeding }\end{array}$ \\
\hline
\end{tabular}

Wang E, Milner R, Navas L, et al. Observer agreement for respiratory signs and oximetry in infants hospitalized with lower respiratory infections. Am Rev Respir Dis 1992;145:106-109. 


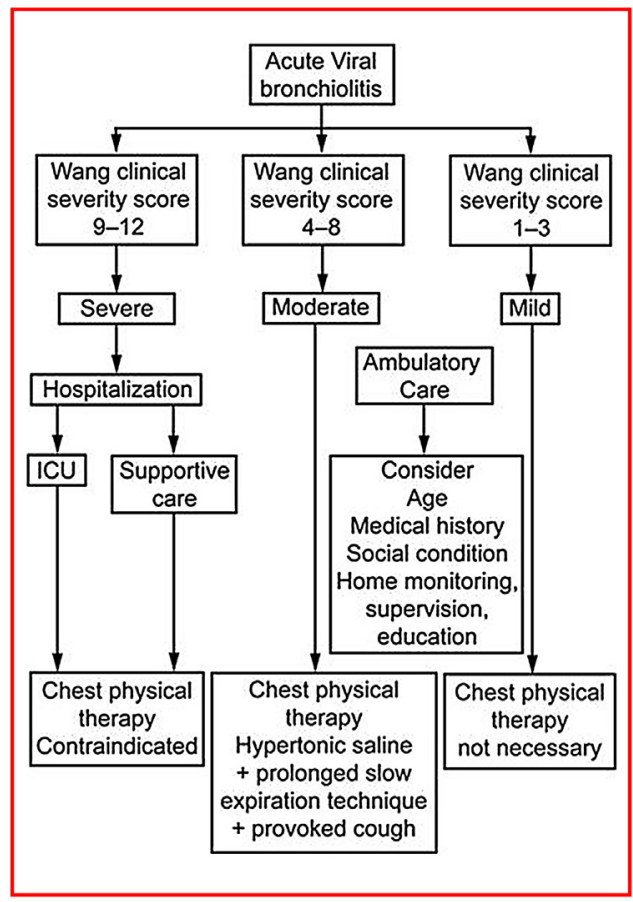

Figure 6. Algorithme de détermination du degré de sévérité de la bronchiolite en relation avec les valeurs du score de Wang. La kinésithérapie respiratoire est indiquée dans la bronchiolite modérée lorsque le score de Wang vaut de 4 à 8 .

Postiaux G, Zwaenepoel B, Louis J. Chest physical therapy in Acute viral bronchiolitis. Un updated review. Resp Care 2013;58:1541-5. (Reproduit avec l'autorisation de Respir Care).

\section{La KR réduit-elle le niveau de gravité de l'obstruction bronchique?}

Il convient de s'interroger sur les mécanismes de clairance des techniques expiratoires lentes appliquées à la désobstruction bronchique dans la BVA. Le SW ne contient qu'une seule variable influençable directement par la KR, à savoir Wh \%. On voit mal en effet comment une manœuvre physique pourrait avoir une action directe sur les trois autres variables du score à savoir la FR, le tirage et l'aspect clinique de l'enfant. Les sibilances constituent le signe dominant de l'obstruction bronchique chez le nourrisson et il est établi que le Wh \% (défini comme la somme des durées des sibilances rapportée à la durée du cycle respiratoire total et exprimée en un pourcentage) est significativement corrélé avec le VEMS chez l'adulte et le grand enfant (étude princeps de Baughman et Loudon en 1982) $[39,40]$ et chez le nourrisson avec la résistance pulmonaire totale (Raw) [41]. Quatre des six études retenues par la Cochrane (Postiaux 2011, Gomez 2012, Sanchez Bayle 2012, Rochat 2012 (dans un sous-groupe) et l'étude postérieure de Van Ginderdeuren 2017 observent une diminution de Wh \% donc du niveau de gravité de l'obstruction bronchique entraînant une diminution du travail ventilatoire et de l'inconfort. Mais que revêt précisément le terme d'obstruction?

\section{La KR de type expiratoire a des effets limités aux bronches proximales}

Les effets de clairance de toute manœuvre expiratoire, quelle qu'en soit l'appellation fonctionnelle ou imagée ou le type de flux produit, restent confinés aux «zones à flux » où sont générées les sibilances, c'est-à-dire aux voies aériennes proximales, trachée et premières bronches, à l'instar de la toux [29]. Dans la majorité du poumon, les flux sont très faibles, voire inexistants [42]. Afin d'obtenir un effet plus « distal » dans l'arbre aérien et pour espérer «traiter » la BVA, à la notion de flux devrait se substituer celle de déflation, ce que tentent de réaliser les expirations lentes par un effet de compression centripète sur le poumon [43] - à l'instar de la pression exercée sur la partie distale d'un tube de pâte dentifrice - en espérant un effet de clairance sur les VAD qui constituent le site principal de l'obstruction bronchopulmonaire. Cet effet sur les VAD est faible étant donné la structure anatomo-fonctionnelle du poumon profond. Il conviendra donc d'envisager la KR « dans » la BVA d'un autre point de vue mécanique, abordé ci-après.

\section{Stricto sensu la KR ne traite pas la BVA}

Une action sur l'arbre aérien proximal attestée par la diminution de Wh \% est observée dans les études. Cette action n'est pas spécifique à la BVA et aucune donnée n'autorise à ce jour d'avancer une action directe sur les VAD au moyen de manœuvres expiratoires à l'exception de quelques observations personnelles portant sur le nombre de craquements de haute fréquence (crépitants). La locution KR dans la BVA est donc inappropriée et l'intitulé de la Cochrane devrait évoluer en KR de l'obstruction bronchique résultant de la BVA. Par contre, le comportement mécanique particulier du poumon profond et quelques études préliminaires invitent à la réalisation de manœuvres inspiratoires profondes et lentes qu'il n'est possible d'obtenir que de manière réflexe chez le nourrisson en réaction à la déflation obtenue par l'ELPr qui entraîne un retour mécanique au niveau d'hyperinflation confortable et qui stimule le réflexe de Hering-Breuer (Fig. 7) [44].

Quelques limites à la KR dans la BVA sont mentionnées par la Cochrane :

- on n'observe pas de réduction de la durée d'hospitalisation : excepté dans l'étude de Van Ginderdeuren (2017) [7] qui montre des délais de guérison significatifs au profit d'expirations lentes en l'occurrence le DAA ;

- l'effet de la KR est transitoire, de faible durée n'excédant pas deux heures : la Cochrane reconnaît cependant l'effet cumulatif intragroupe de jour en jour rapporté par Postiaux et Lens [24] qui laisserait supposer que les séances de KR par l'ELPr prolongent leurs effets durant plusieurs heures (Fig. 8). Ce mécanisme serait intéressant à élucider.

Ajoutons pour mémoire l'expérience chilienne d'un programme public de prise en charge des infections respiratoires chez l'enfant de moins de 5 ans actualisé en $2013[45,46]$. Ce programme a contribué à une réduction significative de la mortalité infantile au Chili. Parmi les principales interventions-actions et dans l'algorithme de ces 


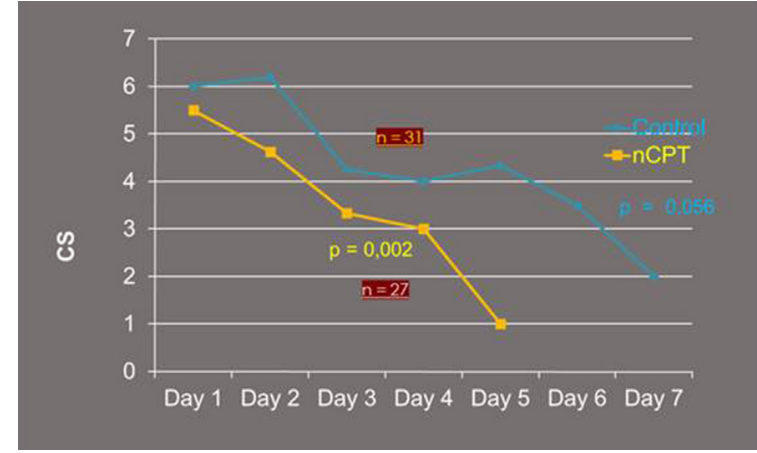

Figure 7. L'assertion « effet transitoire » de la Cochrane est contestable. Si l'on se réfère à l'étude de Postiaux et al. (2011), on observe un effet cumulatif de jour en jour, ce qui signifie qu'une séance de KR prolonge ses effets durant... 24 heures. [Trait jaune $(n=27)$ sur la figure : groupe traité, trait bleu $(n=31)$ : groupe témoin].

Kinésithérapie et bruits respiratoires. Ed DeboechSupérieur 2016:458 et Respir Care 2011 - réf 28.

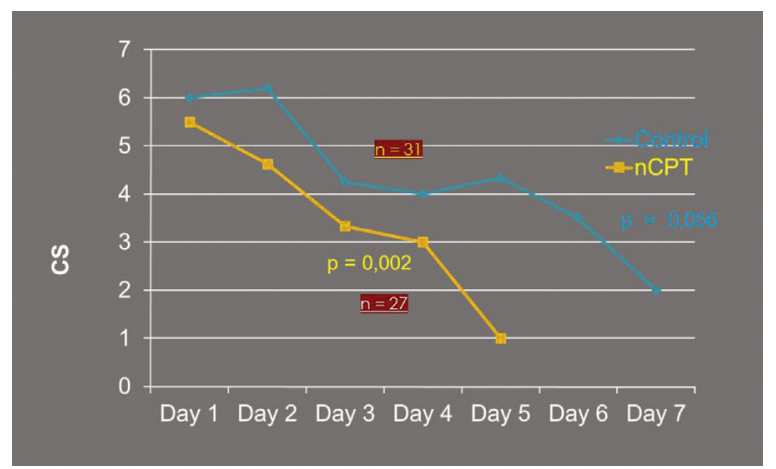

Figure 8. L'assertion " effet transitoire » de la Cochrane est contestable. On observe un effet cumulatif de jour en jour de la KR en intra-groupe, ce qui signifierait qu'une séance de KR prolonge ses effets durant... 24 heures. (Trait jaune [ $n=27]$ sur la figure : groupe traité, Trait bleu [ $n=31]$ : groupe témoin).

(Doc G. Postiaux. Kinésithérapie et bruits respiratoires. Ed Deboecksuperieur 2016. p. 458) et Respir Care 2011 [26].

programmes figure la KR basée sur des techniques expiratoires lentes que le ministère de la Santé chilien classe au niveau de preuve $1+$, recommandation $B$, dans le guide GES des infections respiratoires aiguës de niveau de gravité modérée à légère.

\section{Voies aériennes extrathoraciques}

Des publications récentes rapportent des éléments objectifs de validation de la DRR et de l'éducation parentale à cette technique [47-49]. Ces études montrent des résultats significatifs sur la décongestion nasale, la compliance tympanique mesurés par tympanométrie, la diminution des épisodes d'infections respiratoires basses et de récidives des otites moyennes aiguës (OMA) avec répercussions économiques significatives sur les coûts de santé. La DRR abolit l'usage de l'aspiration nasopharyngée vulnérante, invasive, avec effets secondaires délétères [20].

\section{Conclusion du second exposé «pour»}

La KR ne traite pas la BVA de manière spécifique, mais elle réduit l'obstruction des bronches de premières générations dans la BVA de gravité modérée chez les patients hypersécrétants. Les techniques expiratoires lentes suggèrent une action distale bronchiolaire due à l'inspiration réflexe profonde qui succède à la déflation pulmonaire.

- Arguments pour : avant tout, il faut rappeler que la KR est symptomatique et non pas nosologique, quelle qu'en soit la cause, c'est le caractère sémiologique de l'obstruction bronchopulmonaire qui doit être pris en compte.

- Dans les bronchiolites sévères, comme le recommandent les sociétés savantes, il faut s'abstenir de toute manipulation physique à ce stade de gravité.

- Pour l'AFE qui « entraîne de sérieux effets délétères », on est passé d'une forme initiale assez brutale à des formes plus douces, avec passage d'une accélération vers une augmentation, puis une augmentation lente et enfin une modulation de flux, tendant à rejoindre ainsi un mode expiratoire lent mais la définition de ces termes reste imprécise.

- L'ELPr démontrerait une «amélioration immédiate et transitoire dans la BVA de gravité modérée sans impact sur le décours de la maladie. Des études en ambulatoire sont requises chez les patients infectés par le virus respiratoire syncytial en étudiant l'association de la manœuvre physique à une nébulisation de salbutamol ou de solution saline hypertonique ». Pour la première fois, on fait mention d'une alternative physiothérapique.

- La KR dans la BVA peut être poursuivie à condition de s'appuyer : (1) sur la symptomatologie d'une obstruction de type multifactoriel (œdème, bronchospasme et hypersécrétion des voies aériennes distales, qu'il faut traiter en séquence, et notamment traiter l'œdème avant de procéder à une manœuvre physique, la nébulisation devant précéder les manœuvres physiques) ; (2) sur la validité fonctionnelle d'une manœuvre physique adaptée aux particularités mécaniques du système respiratoire du nourrisson, et (3) en tenant compte des indications ou contre-indications qui reposent sur la sévérité clinique de l'obstruction (la nébulisation iso- ou hypertonique semble mieux tolérée dans la BVA modérée mais peut présenter de sérieux effets adverses dans les formes sévères).

- L'échec des procédures antérieures de la KR doit en partie être attribué à la transposition inappropriée des techniques de l'adulte au jeune enfant. L'ELPr tient compte des caractéristiques mécaniques de l'appareil respiratoire du jeune enfant (trachée compliante, instabilité du thorax, hyperinflation physiologique). 
- La KR est contre-indiquée dans la BVA sévère et inutile dans la BVA légère qui évolue naturellement vers la guérison, mais elle est indiquée dans la BVA modérée, déterminée par un SW de 4 à 8 .

- La séquence logique de la KR comprend nébulisation (NEB) + ELPr + désobstruction rhinopharyngée rétrograde (DRR).

- La KR par des techniques expiratoires lentes permet de diminuer le taux de sibilance (Wh \%), donc le niveau de gravité de l'obstruction bronchique, avec diminution du travail ventilatoire et de l'inconfort.

- Les effets de la KR de type expiratoire sont limités aux bronches proximales et, pour obtenir un effet plus « distal » dans l'arbre aérien et espérer «traiter» la BVA, il faut se référer à la notion de déflation et non de flux, ce que tentent de réaliser les expirations lentes par un effet de compression centripète sur le poumon.

- On ne peut pas affirmer qu'il existe une action directe sur les VAD au moyen de manœuvres expiratoires et, au lieu de parler de KR dans la BVA, il faudrait parler de KR de l'obstruction bronchique résultant de la BVA. Il faut donc effectuer des manœuvres inspiratoires profondes et lentes qu'il n'est possible d'obtenir que de manière réflexe chez le nourrisson en réaction à la déflation obtenue par l'ELPr qui entraîne un retour mécanique au niveau d'hyperinflation confortable et qui stimule le réflexe de Hering-Breuer.

- Des limites à la KR dans la BVA sont mentionnées par la Cochrane : pas de réduction de la durée d'hospitalisation (sauf dans une étude qui montre des délais de guérison significatifs au profit d'expirations lentes, en l'occurrence le DAA) et un effet transitoire de la KR n'excédant pas deux heures, mais il existe un effet cumulatif intragroupe qui laisserait supposer que les séances de KR par l'ELPr prolongent leurs effets durant plusieurs heures.

- Des publications récentes rapportent l'intérêt de la DRR et de l'éducation parentale à cette technique, avec des résultats significatifs sur la décongestion nasale, la compliance tympanique mesurés par tympanométrie, la diminution des épisodes d'infections respiratoires basses et de récidives des otites moyennes aiguës. La DRR rend caduque l'aspiration nasopharyngée vulnérante, invasive, avec ses effets secondaires délétères. plus complexes mais permettraient une évaluation plus convaincante de la KR «dans » la BVA, que laisse entrevoir une action réflexe inspiratoire de l'ELPr sur les VAD. Les effets de la KR sur la durée de l'affection et sur la charge économique induite par les traitements en ambulatoire requièrent des études multicentriques. De récentes études valident les effets des lavages de nez pour la désobstruction des voies aériennes extrathoraciques. L'enseignement de l'auscultation médiate devrait constituer la pierre angulaire de la formation des kinésithérapeutes, comme le suggérait déjà l'ANAES en 2000 sur la base d'un rapport d'expertise [50].

Points essentiels

- L'intérêt de la kinésithérapie respiratoire (KR) dans la bronchiolite virale aiguë du nourrisson (BVA) fait l'objet de vifs débats et une « remise à plat » de cette question semble maintenant nécessaire.

- Les méthodes usuelles de kinésithérapie doivent être abandonnées parce qu'elles ne sont pas validées et parce qu'elles ont des effets secondaires délétères.

- Des techniques expiratoires lentes sont en revanche en partie validées ne présentent aucun effet secondaire délétère, et l'efficacité des lavages de nez pour la désobstruction des voies aériennes extrathoraciques a été établie.

- De larges études multicentriques restent nécessaires pour obtenir des éléments objectifs attestant de l'efficacité des techniques expiratoires lentes sur la désobstruction bronchopulmonaire et sur la réduction de son degré de sévérité à court et moyen termes.

- Les évaluations à venir devraient se fonder sur la physiopathologie d'une obstruction bronchique multifactorielle et sur la sémiologie, dont l'auscultation représente la pierre angulaire.

- Des recherches plus complexes, mais permettant de mieux évaluer la KR dans la BVA, devraient chercher à déterminer les effets des techniques inspiratoires réflexes sur les VAD, notamment sur l'élimination des médiateurs inflammatoires.

- D’autres études, multicentriques, seraient utiles pour déterminer les effets de la KR sur la durée de l'affection et sur la charge économique induite par les traitements en ambulatoire.

\section{Déclaration de liens d'intérêts}

Les auteurs déclarent ne pas avoir de liens d'intérêts.

\section{Références}

[1] Roqué i Figuls $M$, Giné-Garriga $M$, Granados Rugeles $C$, et al. Chest physiotherapy for acute bronchiolitis in paediatric patients between 0- and 24-month-old. Cochrane Database Syst Rev 2016;2:CD004873.

[2] Conférence de consensus sur la prise en charge de la bronchiolite du nourrisson. Arch Pediatr 2001;8:1s-96s. 
[3] Ralston L, Lieberthal AS, Meissner HC, et al. American Academy of Pediatrics. Clinical Practice Guideline: the diagnosis, management and prevention of bronchiolitis. Pediatrics 2014;134:e1474-502.

[4] Baraldi E, Lanari M, Manzoni, et al. Inter-society consensus document on treatment and prevention of bronchiolitis in newborns and infants. It J Pediatr 2014;40:65.

[5] Branchereau E, Branger B, Launay E, et al. État des lieux des pratiques médicales en médecine générale en matière de bronchiolite et déterminants de prises en charge thérapeutiques discordantes par rapport aux recommandations de l'HAS. Arch Pediatr 2013;20:1369-75.

[6] Sterling B, Bosdure E, Stremler-Le Bel N, et al. Bronchiolite et kinésithérapie respiratoire : un dogme ébranlé. Arch Pediatr 2015;22:98-103.

[7] Alauzet F, Banide MC, Pailhes C, et al. Acute cough in infants: impact of national guidelines on medical practices in a French department. Arch Pediatr 2014;21:461-8.

[8] Van Ginderdeuren F, Vandenplas Y, Deneyer M, et al. Effectiveness of airway clearance techniques in children hospitalized with acute bronchiolitis. Ped Pulmonol 2016;52:225-31.

[9] Wang E, Milner R, Navas L, et al. Observer agreement for respiratory signs and oximetry in infants hospitalized with lower respiratory infections. Am Rev Respir Dis 1992;145:106-9.

[10] Paludo C, Zhang L, Lincho CS, et al. Chest physical therapy for children hospitalised with acute pneumonia: a randomised controlled trial. Thorax 2008;63:791-4.

[11] Lukrafka JL, Fuchs SC, Fischer GB, et al. Chest physiotherapy in paediatric patients hospitalised with community-acquired pneumonia: a randomised clinical trial. Arch Dis Child 2012;97:967-71.

[12] Chaves GS, Fregonezi GA, Dias FA, et al. Chest physiotherapy for pneumonia in children. Cochrane Database Syst Rev 2013;9:CD010277.

[13] Bernard-Narbonne F1, Daoud P, Castaing H, et al. Effectiveness of chest physiotherapy in ventilated children with acute bronchiolitis. Arch Pediatr 2003;10:1043-7.

[14] Carroll, Christopher L, Faustino, et al. A regional cohort study of the treatment of critically ill children with bronchiolitis. J Asthma 2016;53:1006-11.

[15] André-Vert J, Gazave M, Goudenege P, et al. Symptômes avant et après kinésithérapie respiratoire : étude prospective auprès de 697 nourrissons du Réseau Kinésithérapie Bronchiolite Essonne. Kinesither Rev 2006;50:25-34.

[16] Gajdos V, Katsahian S, Beydon N, et al. Effectiveness of chest physiotherapy in infants hospitalized with acute bronchiolitis: a multicentre RCT. PLos Med 2010;7:e100035.

[17] Chanelière C, Moreux N, Pracros JP, et al. Rib fractures after chest physiotherapy: a report of 2 cases. Arch Pediatr 2006;13:1410-2.

[18] Chalumeau M1, Foix-L'Helias L, Scheinmann P, et al. Rib fractures after chest physiotherapy for bronchiolitis or pneumonia in infants. Pediatr Radiol 2002;32:644-7.

[19] Gorincour G, Dubus JC, Petit P, et al. Rib periostal reaction: did you think about chest physical therapy? Arch Dis Child 2004;89:1078-9.

[20] Reychler G, Jacques L, Arnold D, et al. Influence de la kinésithérapie respiratoire sur le reflux gastro-œsophagien chez l'enfant. Rev Mal Resp 2015;32:493-9.

[21] Gomes GR, Calvete F, Rosito G, et al. Rhinopharyngeal Retrograde Clearance induces less respiratory effort and fewer adverse effects in comparison with nasopharyngeal aspiration in infants with acute bronchiolitis. Respir Care 2016;61:1613-9.

[22] Postiaux G, Louis J. Feedback letter Cochrane collaboration; 2012.

[23] Postiaux G. La Cochrane review : ce qu'elle dit, n'a pas dit, dira/ne dira pas. Kinesither Rev 2013;13:140-1.
[24] Postiaux G, Lens E. De ladite accélération du flux expiratoire. . où forced is fast (expiration technique-FET). Ann Kinesither 1992;19:411-27.

[25] Postiaux G, Louis J, Labasse HC, et al. Effects of an alternative chest physiotherapy regimen protocol in infants with RSB bronchiolitis. Respir Care 2011;56:989-94.

[26] Rochat I, Leis P, Bouchardy M, et al. Chest physiotherapy using passive expiratory techniques does not reduce bronchiolitis severity: a RCT. Eur Resp J Paediatr 2012;17:457-62.

[27] Gomez EL, Postiaux G, De Medeiros DR, et al. Chest physiotherapy is effective in reducing the clinical score in bronchiolitis: a randomized clinical trial. Bras J Fisioter 2012;16: 241-7.

[28] Sanchez-Bayle, et al. Chest physiotherapy and bronchiolitis in the hospitalized infant. Anales de Pediatria 2012;77:5-11.

[29] Evenou D, Sebban S, Fausser C, et al. Évaluation de l'effet de la kinésithérapie respiratoire avec augmentation du flux expiratoire dans la prise en charge de la première bronchiolite du nourrisson en ville. Kinesither Rev 2017;17:3-8.

[30] Gavriely N, Shee TR, Cugell DW, et al. Flutter in flow-limited collapsible tubes: a mechanism for generation of wheezes. J Appl Physiol 1989;66:2251-61.

[31] Bohadana A, Izbicki G, Kraman S. Fundamentals of lung auscultation. N Engl J Med 2014;370:744-51.

[32] Postiaux G, Lens E. Nomenclature d'auscultation pulmonaire : pourquoi pas un consensus mondial ? Rev Mal Resp 1999;16:1075-89.

[33] Postiaux G. Kinésithérapie et bruits respiratoires. Nouveau paradigme. Ed DeboeckSuperieur; 2016 [445 p. ISBN 978-28073-0307-2].

[34] Zhang L, Mendoza-Sassi RA, Wainwright C, et al. Nebulised hypertonic saline solution for acute bronchiolitis in infants. Cochrane Database Syst Rev 2013;31:CD006458.

[35] Carsin A, Sauvaget E, Bresson V, et al. Early halt of a randomized controlled study with $3 \%$ hypertonic saline in acute bronchiolitis. Respiration 2017;94:251-7.

[36] Angoulvant F, Bellettre X, Milcent K, et al. Effect of nebulized hypertonic saline treatment in emergency departments on the hospitalization rate for acute bronchiolitis: a randomized clinical trial. JAMA Pediatr 2017;171:e171333.

[37] Gajdos V, Beydon N, Bommenel L. Inter-observer agreement between physicians, nurses and respiratory therapists for respiratory clinical evaluation in bronchiolitis. Pediatr Pulmonol 2009;44:754-62.

[38] Postiaux G, Zwaenepoel B, Louis J. Chest physical therapy in acute viral bronchiolitis: an updated review. Respir Care 2013;58:1541-5.

[39] Baughman RP, Loudon RG. Quantitation of wheezing in acute asthma. Chest 1984;86:718-22.

[40] Sanchez I, Powell RE, Pasterkamp H. Wheezing and airflow obstruction during methacholine challenge in children with cystic fibrosis and in normal children. Am Rev Respir Dis 1993;147:705-9.

[41] Postiaux G, Ladha K, Gillard C, et al. La kinésithérapie respiratoire du petit enfant (<24 mois) guidée par l'auscultation pulmonaire. Rev Fr Allergol 1997;37:206-22.

[42] Postiaux G. Kinésithérapie du poumon profond. Bases mécaniques d'un nouveau paradigme. Rev Mal Respir 2014;31:552-67.

[43] Postiaux G, Lens E, Alsteens G. L'Expiration lente totale glotte ouverte en decubitus latéral (ELTGOL) : nouvelle manœuvre pour la toilette bronchique objectivée par vidéo bronchographie. Ann Kinesither 1987;14:341-5.

[44] Lanza F, Wandalsen G, Bianca AC, et al. Prolonged slow expiration technique (PSE): description of pulmonary alterations in infants. Respir Care 2011;56:1930-5.

[45] Girardi G, Astudillo P, Zúñiga F. El programa IRA en Chile: hitos e historia. Rev Chil Pediatr 2001;72:292-300. 
[46] Ministerio de, Salud. Guía Clínica Infección Respiratoria Aguda Baja de Manejo Ambulatorio en Menores de 5 años. MINSAL; 2013.

[47] Alexandrino AS, Santos R, Melo C, et al. Caregivers'education vs rhinopharyngeal clearance in children with upper respiratory infections: impact on children's health outcomes. Eur J Pediatr 2017;363:1-9.

[48] Alexandrino AS, Dos Santos R, De Melo MC, et al. Designing and evaluating a health education session on respiratory infections addressed to caregivers of children under three years of age attending day-care centres in Porto, Portugal: a communitybased intervention. Eur J Gen Pract 2017;23:43-50.

[49] Alexandrino AS, Santos R, Melo C, et al. Impact of caregivers'education regarding respiratory infections on the health status of day-care children: a RCT. Fam Pract 2016;33:476-81.

[50] Postiaux G. Quelles sont les techniques de désencombrement bronchique et des voies aériennes supérieures adaptées chez le nourrisson ? Rapport d'expertise Conférence de Consensus sur la Bronchiolite du Nourrisson. Paris 21 septembre 2000. Arch Pediatr 2001;8:117-25. 